\title{
Hemangiopericytoma- A solitary tongue lesion
}

\author{
Sangeeta P. Wanjari ${ }^{1, *}$, Panjab V. Wanjari ${ }^{2}$ \\ Professor \& HOD, Dept. of Dentistry, ${ }^{1}$ BKL Walawalkar Rural Medical College, Ratnagiri, Maharashtra, ${ }^{2}$ Modern Dental \\ College and Research Centre, Gandhinagar, Indore, Madhya Pradesh, India
}

*Corresponding Author:

Email: drsangeetawanjari@gmail.com

\begin{abstract}
Hemangiopericytoma (HPC) is a mesenchymal tumour that is usually benign, but has definite malignant counterpart. 16-25 \% cases of all reported HPC's are observed involving Head and Neck region. We report a case of HPC on the left lateral border of tongue. The histopathology features revealed increased cellularity, vascularity with some staghorn pattern. Observed nuclear pleomorphism and vesicular as well as hyperchromatic nuclei suggest an intermediate grade characterized by unpredictable clinical behaviour. Therefore long follow up is essential. No local recurrence or distant metastasis was noticed in a 5 year followup period.
\end{abstract}

Keywords: Hemangiopericytoma, Vascularity, Staghorn.

\section{Introduction}

Hemangiopericytoma (HPC) has been described as a distinct entity in 1942 by Stout and Murray. ${ }^{1}$ Pericytes-modified smooth muscle cells, located around the blood vessels were considered to be cell of origin for HPC's. ${ }^{2,3}$ The pericytes, a cell type described by Zimmermann in 1923, are located outside the reticulin sheath of the endothelium. ${ }^{2-4}$

Soft tissues of the upper and lower extremities and retro-peritoneum are most commonly affected by HPC's. Around 16-25\% cases involving head-neck region are reported. In head neck area areas involved are; orbit, nasal sinus tract, salivary gland (parotid, submandibular, sublingual), tongue, lip, maxilla, mandible, floor of mouth, hard palate, buccal mucosa, retromolar gingiva, parapharyngeal spaces, thyroid and $\operatorname{larynx} .^{3,4}$

Oral HPC occurs most often in adults, with average age of 52 years $^{2}$, without any gender predilection. ${ }^{1,4,5}$ The biological behaviour of HPC is unpredictable. The features like cellular pleomorphism, mitoses $>4$ per high-power fields, high proliferation index, and necrosis represent the malignant forms. ${ }^{4,5}$ Here we report a case of HPC on the left lateral border of tongue with an uneventful 5 year follow up.

\section{Case Report}

A 48 year male, reported to the department of Oral Pathology with a chief complaint of painless mass on left-side of tongue, since $1-1{ }^{1 /}{ }_{2}$ months; with occasional bleeding. Intra oral examination revealed a single well defined oval shaped, pedunculated growth on left lateral border; $4 \mathrm{~cm}$ posterior to the tip of tongue; measuring approximately $2 \times 1.5 \mathrm{~cm}$ in diameter. Growth revealed mild lobulations; having erythematous, glistening overlying mucosa with tiny red spots. Focal greyish white areas were evident on one side towards the stalk. The growth had constricted neck with approximately 6 $\mathrm{mm}$ long and $3 \mathrm{~mm}$ wide stalk (Fig. 1). Palpation revealed growth to be non tender, firm in consistency and was slightly bleeding on manipulation. However no pulsations were felt with the lesion. Provisional diagnosis of pyogenic granuloma with differential diagnosis of fibroma, schwannoma was considered.

The lesion was completely removed by wide surgical resection. Histopathologic examination revealed excessively vascular connective tissue stroma having numerous single endothelially lined blood vessels of variable shape and size, some of which showed characteristic staghorn pattern. Stroma appeared densely cellular around blood vessels and revealed proliferation of oval and spindled shaped cells with large vesicular nuclei. However at places hyperchromatic nuclei and nuclear pleomorphism too was evident (Fig. 2). Histopathologic features suggested HPC (intermediate grade) a final diagnosis. The patient had a normal postoperative course of healing, and 5 years follow up remains asymptomatic, without signs of recurrence or metastasis.

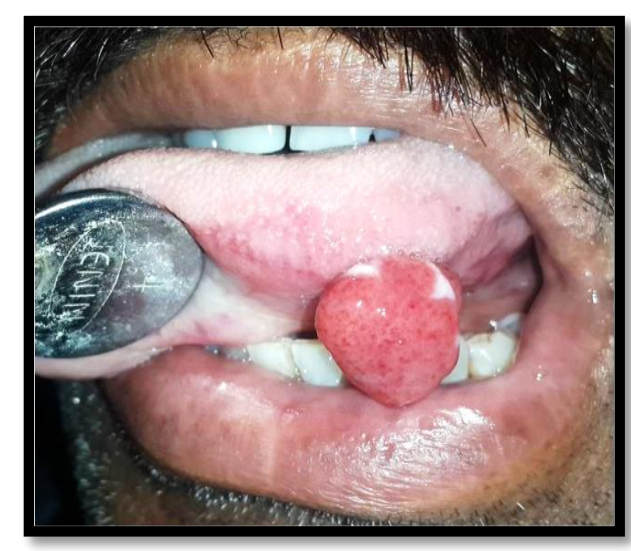

Fig. 1: Clinical presentation of the lesion on left lateral border of the tongue 

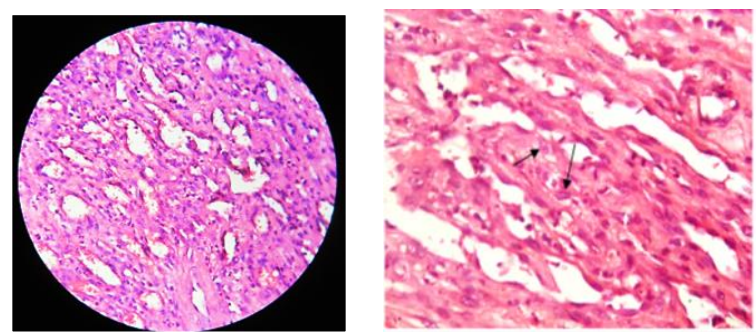

Fig. 2: Histopathology reveals numerous thin-walled branching vessels with 'staghorn' pattern and dense cellular stroma around them (arrows reveal vesicular nuclei)

\section{Discussion}

HPC is a rare neoplasm, affecting soft tissues of upper and lower extremities as well as head-neck region including salivary glands and oral cavity. It was thought to arise from pericytes, the modified smooth muscle cells present outside the walls of capillaries and arterioles. ${ }^{2,3}$ However, it is not clear that the neoplasm actually derives from these cells, as most of these adulthood tumours immunohistochemically stain non specifically for vimentin and CD34, but not for actin or other myoid markers. ${ }^{5,6}$ Various other etiological factors have also been suggested based on the medical history and clinical findings as hypertension, hormonal or metabolic imbalance, and trauma. ${ }^{4}$ Moreover, Chromosomal translocations $\mathrm{t}(12,19)$ and $\mathrm{t}(13,22)$ observed in lesional cells suggest genetic etiology. ${ }^{1}$

HPC occurs as a localized mass; with variable size ranging from 1 to $20 \mathrm{~cm}$ in diameter ${ }^{4}$. Oral HPC reveals variable growth pattern. It could be either rapidly enlarging or slow growing, red to bluish; often well circumscribed and asymptomatic mass. ${ }^{1,3}$ It may be sessile or pedunculated and may demonstrate surface lobularity or telangiectasis ${ }^{1}$. Present case too revealed pedunculated well localized erythematous growth with mild lobulations.

Histopathology of HPC exhibits moderate to dense cellular stroma around thin walled dilated vessels lined with flat endothelial cells. Antlers like branching vessels are prominently observed; which are named as staghorn pattern. ${ }^{4,7}$

The clinical behaviour of HPC varies from case to case. Histological demonstration of cellular density, cellular and nuclear pleomorphism, mitotic activity and necrosis classify HPC as low-grade, intermediate grade, or high-grade. ${ }^{3-5}$ However, prediction of biological behaviour of HPC appears difficult as histologic criteria are imprecise for distinction between benign and malignant counterpart ${ }^{3}$. Because of the rarity and unpredictable biological behaviour of these tumours, effective management remains controversial. Wide surgical resection remains the treatment of choice for small localized $\mathrm{HPC}^{3}$. Although tumour is relatively radioresistant; recent studies suggest that radiation therapy may be useful in some situations. ${ }^{3,8}$ In case of incomplete surgical removal, postoperative radiation therapy has been recommended. However; role of chemotherapy in case of locally non-operative HPC has not been clearly demonstrated. ${ }^{3}$

In 20 to $57 \%$ HPC cases, recurrence had been reported after 17 months from initial treatment. ${ }^{4}$ However; after a disease-free interval of 3 years, Enzinger and Smith had reported recurrence in 7 out of 106 patients.

As histological features are poorly correlated with the clinical behaviour of the tumour; prognosis of HPC is unpredictable. ${ }^{4} 12$ to $56 \%$ of high grade malignant HPCs have been reported to metastasize to lymph node, bone, lung, liver and pancreas. ${ }^{3,4,8}$ Metastases usually occur within 5 year after initial diagnosis and are rare after 10 years. ${ }^{4}$

Because of the high recurrence rate and difficulty in predicting the clinical behaviour of HPC, long-term follow-up care is recommended..$^{5}$

\section{Conclusion}

HPC is a rare vascular tumour, with variable clinical presentation. Its prediction of biological behaviour appears difficult as histologic criteria are imprecise for distinction between benign and malignant counterpart. The treatment of choice remains wide surgical resection. As it demonstrates definite malignant potential, long-term follow-up is necessary even after radical resection; because recurrence or metastasis may be delayed by many years.

\section{References}

1. Benign and Maligmant Tumours of the Oral cavity. In: Shafer's Textbook of Oral Pathology. R. Rajendran, B Shivpathasundharam, $6^{\text {th }}$ ed. Elsevier: Noida 2009:162-3.

2. Blood Vessels. In: Robbins Basic Pathology of Tumours. Kumar V, Abbas AK, Aster JC. $9^{\text {th }}$ ed. Elsevier Saunders: Philadelphia 2013;362.

3. Angiero F, Signore A, Benedicenti S. Hemangiopericytoma/Solitary Fibrous Tumour of the Oral Cavity. Anticancer Res 2011;31,719-24.

4. Maresi E, Tortorici S, Campione M, Buzzanca ML, Burruano F, Mastrangelo F et al. Case Report: Hemangiopericytoma of the Oral Cavity after a Ten-year Follow-up. Annals of Clinical \& Laboratory Science 2007;37(3), 274-9.

5. Vascular Tumours. In: Diagnostic Histopathology of Tumours. Fletcher CDM, $4^{\text {th }}$ ed. Elsevier Saunders: Philadelphia 2013;77-80.

6. Diseases of Individual Organ Systems. In: Rubin's Pathology- Cliniocopathologic Foundationof Medicine. Strayer DS, Rubin E, Saffitz JE, Schiller AL. $7^{\text {th }}$ ed. Wolters Kluwer: Philadelphia 2015;618.

7. Veltrini VC, Etges A, Magalhães MH, de Araújo NS, de Araújo VC. Solitary fibrous tumour of the oral mucosamorphological and immunohistochemical profile in the differential diagnosis with hemangiopericytoma. Oral Oncol 2003;39(4),420-6.

8. Backwinkel KD, John A, Diddams JA. Hemangiopericytoma- Report of a case and comprehensive review of the literature. Online library.Wiley.com/action; https://doi.org/10.1002/10970142(197004)25:4<896::AID-

CNCR2820250423>3.0.CO;2-N. 